\title{
Research Projects on Jaw Function and Occlusion Published in 2006 by Members of the Japan Prosthodontic Society: A Review
}

\author{
Taihiko Yamaguchi, DDS, PhD \\ Department of Temporomandibular Disorders, Center for Advanced Oral Medicine, \\ Hokkaido University Hospital, Sapporo, Japan
}

Key words: masticatory function, temporomandibular disorders, shortened dental arch, sports dentistry, mandibular movement

\section{Introduction}

This article reviews the findings of 5 original studies in the areas of occlusion and jaw function published in the Journal of the Japan Prosthodontic Society (J Jpn Prosthodont Soc; Nippon Hotetsu Shika Gakkai Zasshi in Medline/PubMed) issued in Volume 50, 2006. In these studies, masticatory function of removable denture wearers and temporomandibular disorders patients, clinical states of shortened dental arch, activity of masticatory muscle during exerting back muscle strength and a newly developed jaw motion tracking device were examined. These studies were considered to contribute toward advances in prosthodontic researches and practice.

\section{Masticatory function}

In prosthodontic practice, it is necessary to estimate treatment effects by evaluating mastication ability before and after prosthodontic treatments. Various methods for evaluating mastication ability have been reported. As food comminution tests, sieve methods ${ }^{1,2}$ and quantification of glucose extraction after gummy-jelly chewing $^{3}$ were used. Mixing ability tests using chewing gum ${ }^{4}$ and wax $^{5}$ were also undertaken. Electromyograph(EMG) ${ }^{6}$ and jaw movement analysis ${ }^{7}$ were substituted for food comminution tests. Ability to form food bolus was analyzed us-

Corresponding to: Dr Taihiko Yamaguchi

Department of Temporomandibular Disorders, Center for

Advanced Oral Medicine, Hokkaido University Hospital

West 7 North 13 Kita-ku, Sapporo 060-8586, Japan

Tel\&Fax: +81-11-706-4856

E-mail: taihiko@den.hokudai.ac.jp ing videofluorography. ${ }^{8}$ As subjective tests, questionnaires on food intake ability were used. ${ }^{9}$

Honma et $\mathrm{al}^{10}$ applied a new method utilizing number of chewing strokes by visual observation to denture wearers for evaluating the mastication ability focusing on bolus formation. One hundred and twenty-five subjects (71 males and 54 females) using removable dentures and receiving follow-up were selected. The subjects were categorized by Eichner's classification, and investigated for the effect of prosthetic treatment by counting the number of chewing strokes until the first swallow of rice crackers. It was clarified that the number of chewing strokes until the first swallow counted by visual observation agreed well with the number of chewing strokes counted by electromyogram wave of masseter muscle, laryngeal motion, and swallowing sound (Fig 1). Using the method of counting chewing strokes by visual observation, the effect of prosthetic treatment was evaluated. A significant difference in the number of chewing strokes without dentures was observed in the Eichner A group, B group, and $\mathrm{C}$ group, while there was no significant difference in the number of chewing strokes with dentures among the three groups. The use of removable dentures led to a significant decrease in the number of chewing strokes. The rate of improvement in the number of chewing strokes (the ratio of the number of chewing strokes until the first swallow with dentures to that without dentures) and the difference in the number of chewing strokes increased significantly in the order of the Eichner A, B, and C group. These findings indicated that the effects of prosthetic treatment can be quantitatively evaluated by counting the number of chewing strokes of rice cracker. Moreover, it was suggested that the condition of prosthetic treatment can be evaluated by analyzing the improvement rate in the number of chewing strokes. The method used in this study was easy and objective, and is expected to be clinically useful for assessment of prosthodontic treatments. 


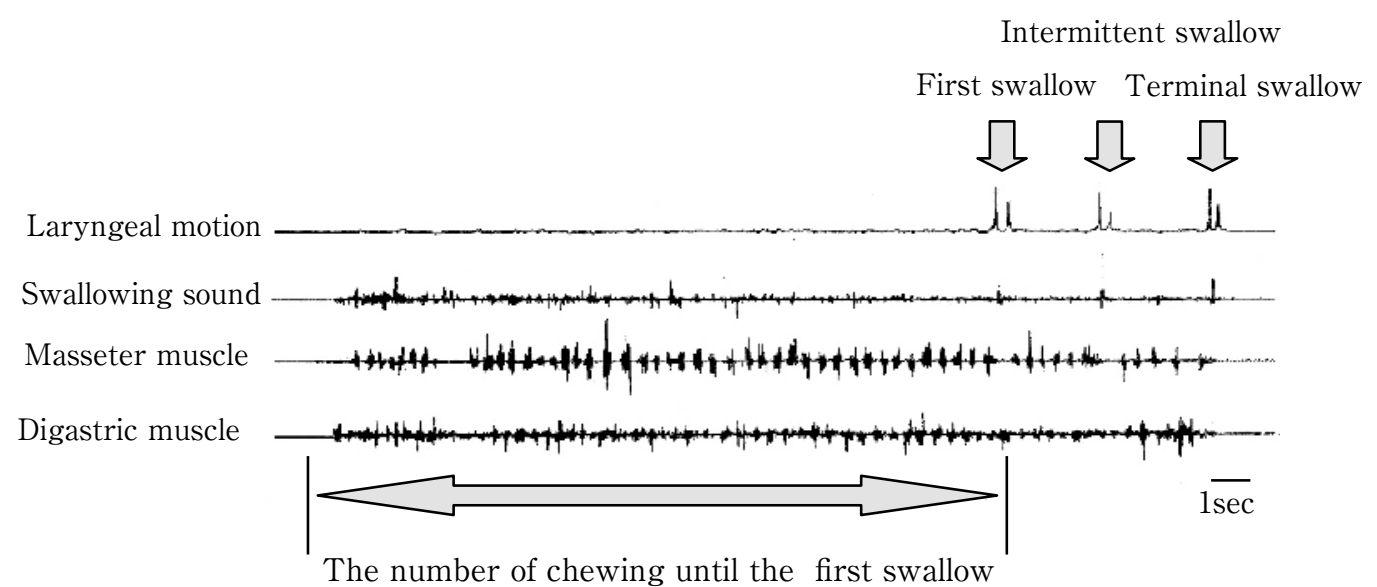

Fig. 1 The observation on the number of chewing strokes and swallowing by EMG, laryngeal motion, and swallowing sound. ${ }^{10}$ (Honma et al)

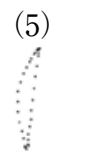

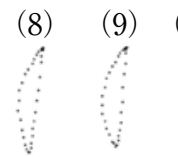

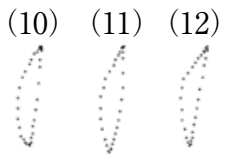

(13)

(14)
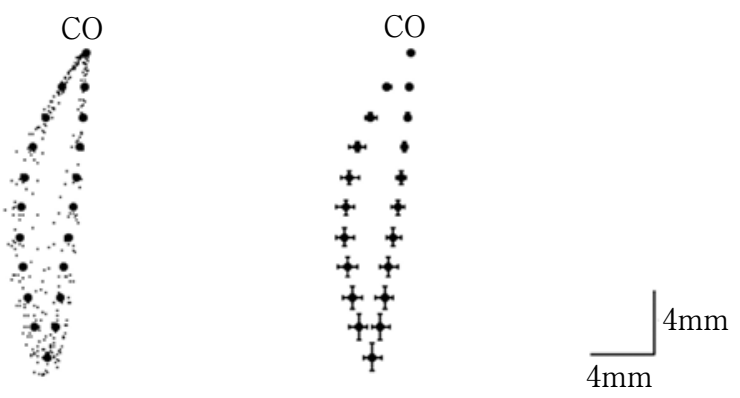

[ Right side chewing ]

Fig. 2 Each cycle from the fifth cycle to the fourteenth cycle of chewing (right side), overlapping of the ten cycles and its average path and standard deviation as seen frontal plane. ${ }^{12}$ (Mizuuchi et al) CO: centric occlusion.

\section{Temporomandibular disorders}

Temporomandibular disorders (TMD) are thought to be involved in disturbance of masticatory function. ${ }^{11}$ However, objective evaluation of masticatory function of TMD patients has not been reported sufficiently.

Mizuuchi et al $^{12}$ attempted to evaluate the masticatory function in TMD objectively by means of a comprehensive method using EMG, jaw movement analyses and a food comminution test. The quantitative indicators of muscular activity, stability of movement, and masticatory efficiency during mastication were compared among the following 3 groups: 10 healthy sub- jects, 10 patients with arthrogenous TMD, and 10 patients with myogenous TMD. For the evaluation of muscular activity, the integral value of the masseter muscular activity was calculated. The average mandibular path and the standard deviations of each analyzed point were calculated horizontally and vertically (Fig.2) and 3 indicators of the stability of path were calculated according to the method of analysis used in their previous study. ${ }^{13}$ Four indicators of rhythm, i.e. coefficient of variation of duration of opening, closing and occlusal phases, and of cycle time were also calculated. For stability of movement, a comprehensive indicator was established after a principal component analysis using the data from the 7 indicators of stability of path and rhythm. For an assessment of masticatory efficiency, the amount of glucose extraction after gummy-jelly chewing was measured by a blood glucose testing device. The cumulative value of the masseter muscular activity and the amount of glucose extraction after gummy-jelly chewing were higher in the healthy subject group than in the groups with arthrogenous and myogenous TMD, and these differences between the healthy subject group and the other 2 groups were statistically significant. On the other hand, the value of the comprehensive indicator was significantly smaller in the healthy subject group than in the groups with arthrogenous and myogenous TMD. These findings suggested that the masticatory function in TMD patients is considerably inferior in comparison to that in the healthy subjects. 


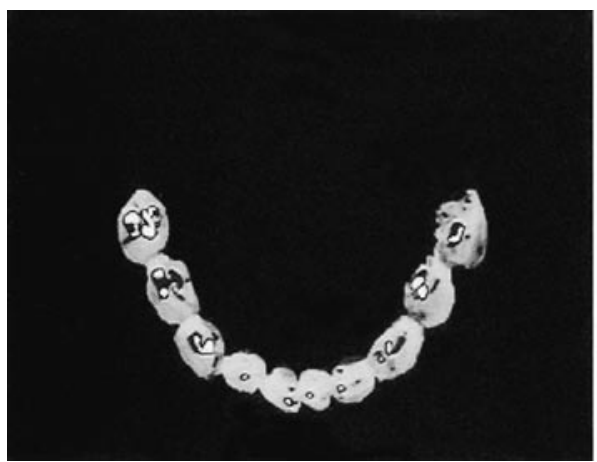

SDA Case

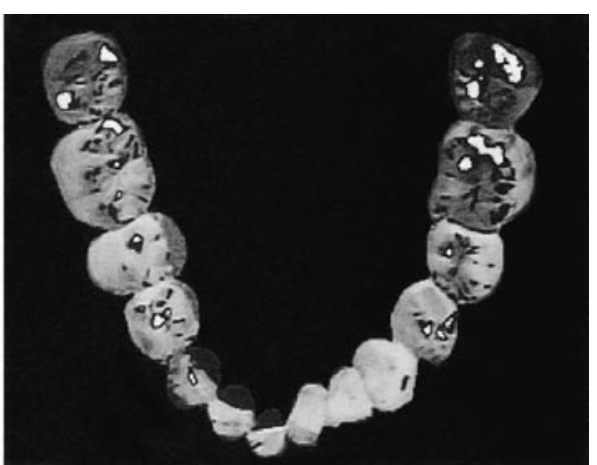

CDA Case

Fig. 3 Examples of add-picture in each group. ${ }^{15}$ (Arai et al)

\section{Shortened dental arch}

Recently, there have been reports in favor of avoiding prosthodontic intervention as Shortened Dental Arch (SDA) in Northern Europe. ${ }^{14}$ However, many of these reports were conducted through interviews and questionnaires, and the evaluation of the results lacked objectivity. It is thought to be necessary to establish an appropriate treatment guideline for SDA based on evident data using diversified and objective evaluation.

Arai et $\mathrm{al}^{15}$ analyzed the clinical state of SDA in 7 patients who had had the free-end missing on both sides of the lower dental arches for over 5 years. As the complete dental arch (CDA) group, 7 patients who had no clinical defects in their masticatory function, and who had no missing teeth other than the third molar, were selected. Occlusal contact areas for the mandible premolar measured by add-picture method (Fig. 3), center of force and occlusion time by means of T-Scan II $^{\circledR}$ system(Tekscan, Inc. USA), and interdentium measured with steel contact gauges were compared between the SDA and CDA groups. It was observed that the occlusal contact area of the mandible premolar of SDA was significantly larger than that of CDA and the center of force in the SDA group was significantly forward. The occlusion time in the SDA group had a tendency toward prolongation. Interdentium of $\overline{32} 23$, $\overline{4354}$ and $\overline{54 \mid 45}$ of SDA were significantly wider. They concluded that there were differences in the clinical parameters between the SDA and CDA in this study. This study partially clarified the cross-sectional features of SDA. In order to establish treatment guidelines for SDA, further studies on longitudinal observation of the masticatory function in SDA subjects are expected.

\section{Sports dentistry}

It is thought that there is close relationship between body exercise and jaw functions. The lower jaw is considered to be fixed during body exercise. However, its mechanism remains to be elucidated.

Asano et $\mathrm{al}^{16}$ investigated masticatory muscle activity of 9 healthy dentulous subjects during exerting back muscle strength. The maximum back strength was measured with a back-dynamometer. EMG activities of the temporal, masseter and digastric muscles during exertion of the back, mastication of peanuts and maximum voluntary contraction were measured (Fig. 4). Relative percentages for the maximum voluntary contraction of each muscle were calculated and compared. The relative percentages of muscle activities of the temporal, masseter and digastric muscles during the exertion of the back muscles were $32.1 \%, 26.4 \%$ and $97.4 \%$, respectively, and those during the mastication of peanuts were $40.7 \%, 36.0 \%$ and $17.3 \%$, respectively. These findings suggested that the strong clenching force generated by the jaw-closing muscles does not arise during the exertion of the back. On the other hand, the muscle activity of the digastric muscle was approximately $100 \%$ and it was suggested that the digastric muscles play a key role in fixing the mandible. People exert the strength of the back not only in sports but also in their daily life. This study demonstrates the stronger involvement of the digastric muscles in fixation of the mandible during exercise than strong clenching by jaw closing muscles. The results are of interest in terms of the mandibular position, occlusal contact and the load on the temporomandibular joint (TMJ) since there is no wide mouth opening. 

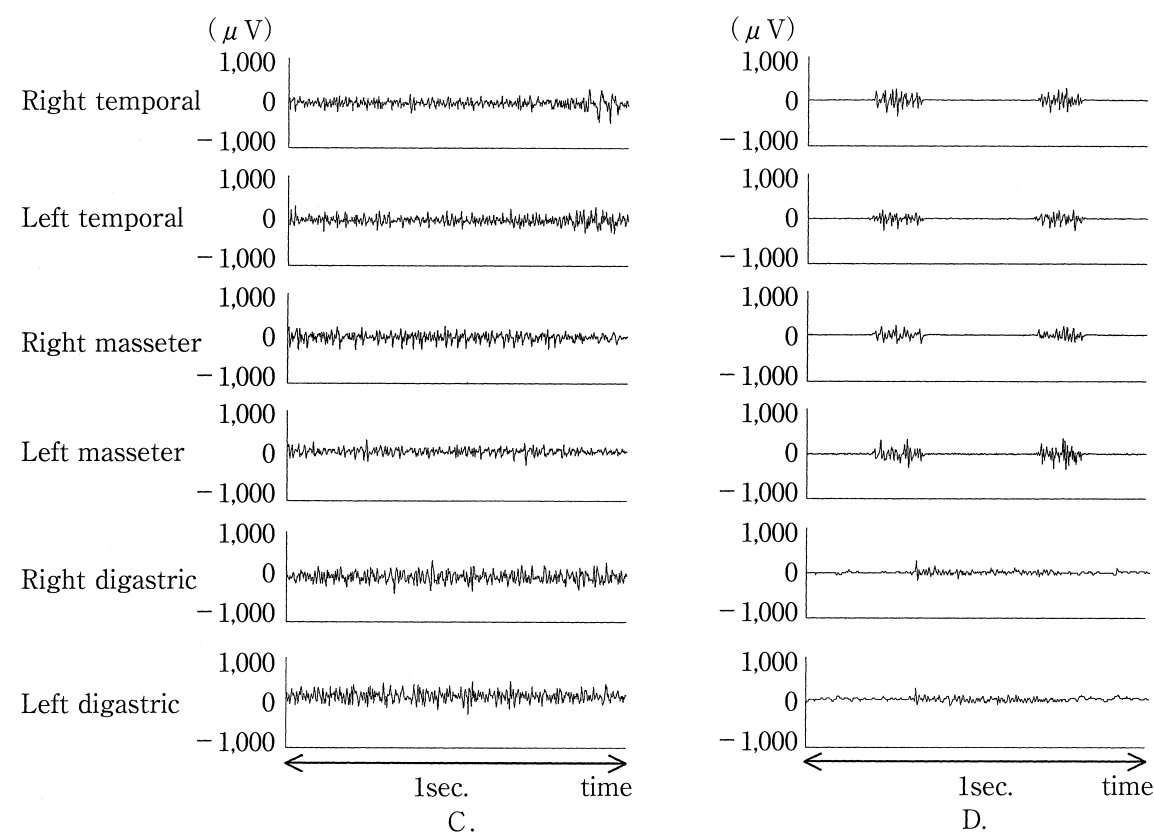

Fig. 4 Electromyographic patterns. ${ }^{16}$ (Asano et al) C: exertion using back strength, D: mastication of peanuts.

Lateral occlusalposition

Right Left

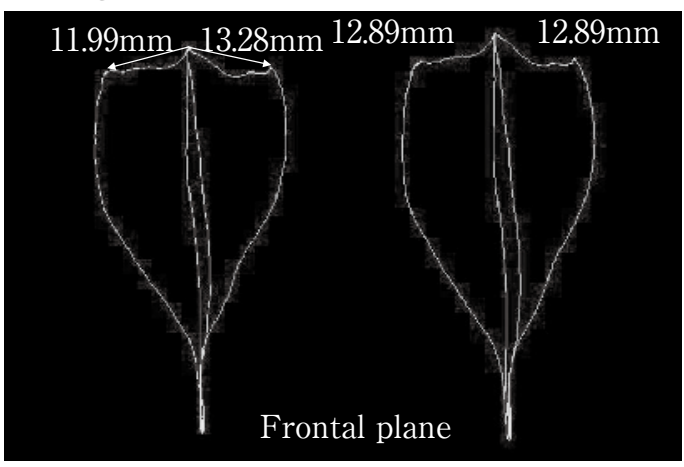

Digital system

Optical system

Fig. 5 An example of border mandibular movements in frontal plane with two jaw tracking devices. ${ }^{20}$ (Ogawa et al)

\section{Jaw motion tracking device}

Worldwide, several jaw movement analyzing devices capable of recording with 6 degrees of freedom have been developed. In Japan, MMJI-E (SHOFU Co., Kyoto, Japan) ${ }^{17}$, TRI-MET (TOKYO SHIZAISHA Co., Tokyo, Japan ${ }^{18}$ and Gnathohexagraph JM-1000 (Ono Sokki Co., Tokyo, Japan $)^{19}$ have been developed and are available on the market. These jaw movement analyzing devices have been applied to dental practice and have contributed to the elucidation of features of various jaw functions.

Ogawa et $\mathrm{al}^{20}$ recently developed a new jaw tracking device to achieve jaw movement analyses with simpler operation and lower cost and examined its potential for clinical applications. The new device is an optical system using position sensitive detector (PSD). The weights of upper and lower face bow attached to dental arch are $30 \mathrm{~g}$ and $35 \mathrm{~g}$, respectively. Each face bow has 3 light emitting diodes (LED). In evaluation of measurement precision in the 3 -dimensional space $(170 \times 200 \times 200 \mathrm{~mm})$, the root mean square (RMS) error of position measurement between the true value and the calculated coordinate point was $0.178 \mathrm{~mm}$ with the new optical type device and $0.163 \mathrm{~mm}$ with a conventional digital system type device (MM-JI-E). In simultaneous measurement by the new device and MM-JI-E, jaw motions (open-close, sagittal border and frontal border movements) of 3 healthy female subjects were analyzed. At the anterior reference point, the error of the measurement with the new optical type device in comparison with MM-JI-E was maximal at $0.8 \mathrm{~mm}$ and minimal at $0.1 \mathrm{~mm}$ and the traced pathway was similar to that obtained with MM-JI-E (Fig. 5). These findings suggested that the new device has potential of clinical use for analyzing in anterior area near the incisor. As for the pathways at the condylar point, relatively rougher traces that fluctuated in the vertical direction were recorded with the new optical type device. The authors thought that resolution in the posterior area near the condyle 
became lower because the area was apart from the LEDs, and they mentioned that further studies on the resolution are needed.

\section{References}

1. Manly RS, Braley LC. Masticatory performance and efficiency. J Dent Res 29: 448-462, 1950.

2. Ohara A, Tsukiyama Y, Ogawa T et al. A simplified sieve method for determining masticatory performance using hydrocolloid material. J Oral Rehabil 30: 927-935, 2003.

3. Umehara A, Nokubi K, Yasui S et al. The method of improving the evaluation of masticatory efficiency in complete denture wearers by using the testing gumi-jelly. J Jpn Prosthodont Soc 41: 850-855, 1997.

4. Hirano S, Takahashi Y, Watanabe I et al. Application of color-changeable chewing gum for measuring masticatory performance of complete denture wearers. J Jpn Prosthodont Soc 45: 730-736, 2001.

5. Satoh H, Fueki K, Sueda S et al. A new and simple method for evaluating masticatory function using newly developed artificial test foods. J Oral Rehabil 30: 68-73, 2003.

6. Kapur KK. Studies of biologic parameters for denture design. Part I. Comparison of masseter muscle activity during chewing of crisp and soggy wafers in denture and dentition groups. J Prosthet Dent 33: 242-249, 1975.

7. Woelfel JB, Hickey JC, Allison ML. Effect of posterior teeth form on jaw and denture movement. J Prosthet Dent 12: 922-939, 1962.

8. Kato K, Kohno S, Arai E et al. Denture prosthetic treatment by assistance of videofluorograghy. J Jpn Prosthodont Soc 44: 625-632, 2000.

9. Satoh Y, Ishida E, Minagi S et al. The aspect of dietary intake of full denture wearers. J Jpn Prosthodont Soc 32: 774-779, 1988.
10. Honma W, Kohno S, Sakurai N et al. Evaluation of prosthetic treatment using number of chewing strokes until swallowing of water absorbing rice crackers. J Jpn Prosthodont Soc 50: 219-227, 2006.

11. Sessle BJ. Biological adaptation and normative values. Int J Prosthodont 16(Supplement): 72-73, 2003.

12. Mizuuchi K, Shiga H, Kobayashi Y. Objective evaluation of masticatory function in temporomandibular disorders patients. J Jpn Prosthodont Soc 50: 405-413, 2006.

13. Shiga H, Kobayashi Y. An objective evaluation of masticatory function by analysis of masticatory movements. J Jpn Prosthodont Soc 34: 1112-1126, 1990.

14. Sarita PT, Kreulen CM, Witter DJ et al. A study on occlusal stability in shortened dental arches. Int J Prosthodont 16: 375-380, 2003.

15. Arai K, Tsurumi A, Takao T et al. Comparison of shortened dental arches with complete dental arches by clinical examinations. J Jpn Prosthodont Soc 50: 26-34, 2006.

16. Asano T, Kawara M, Suzuki H et al. Masticatory muscle activity during exertion of the back. J Jpn Prosthodont Soc 50: 45-53, 2006.

17. Fujimura T, Bando E. The development of a digital type jaw movement analyzer. J Jpn Prosthodont Soc 35: 830-842, 1991.

18. Kohno S. Application of the TRIMET in scientific research of stomatognathic functions. J Jpn Prosthodont Soc 42: 913-920, 1998.

19. Tokiwa H, Kuwahara Y. Clinical examination for stomatognathic functions - By using Gnathohexagraph—. J Jpn Prosthodont Soc 42: 902-912, 1998.

20. Ogawa T, Shigeta Y, Ando E et al. Application of a jaw motion tracking device that measures six degrees of freedom using optoelectronic. J Jpn Prosthodont Soc 50: 210-218, 2006. 